\title{
Determination of the unknown boundary condition of the inverse parabolic problems via semigroup method
}

\section{Ebru Ozbilge*}

\section{"Correspondence:}

ebru.ozbilge@ieu.edu.tr

Department of Mathematics,

Faculty of Science and Literature,

Izmir University of Economics,

Sakarya Caddesi, No. 156, Izmir,

Balcova 35330, Turkey

\begin{abstract}
In this article, a semigroup approach is presented for the mathematical analysis of inverse problems of identifying the unknown boundary condition $u(1, t)=f(t)$ in the quasi-linear parabolic equation $u_{t}(x, t)=\left(k(u(x, t)) u_{x}(x, t)\right)_{x}$, with Dirichlet boundary conditions $u(0, t)=\psi_{0}, u(1, t)=f(t)$, by making use of the over measured data $u\left(x_{0}, t\right)=\psi_{1}$ and $u_{x}\left(x_{0}, t\right)=\psi_{2}$ separately. The purpose of this study is to identify the unknown boundary condition $u(1, t)$ at $x=1$ by using the over measured data $u\left(x_{0}, t\right)=\psi_{1}$ and $u_{x}\left(x_{0}, t\right)=\psi_{2}$. First, by using over measured data as a boundary condition, we define the problem on $\Omega_{T_{0}}=\left\{(x, t) \in R^{2}: 0<x<x_{0}, 0<t \leq T\right\}$, then the integral representation of this problem via a semigroup of linear operators is obtained. Finally, extending the solution uniquely to the closed interval $[0,1]$, we reach the result. The main point here is the unique extensions of the solutions on $\left[0, x_{0}\right]$ to the closed interval $[0,1]$ which are implied by the uniqueness of the solutions. This point leads to the integral representation of the unknown boundary condition $u(1, t)$ at $x=1$.
\end{abstract}

\section{Introduction}

Consider the following initial boundary value problem for quasilinear diffusion equation:

$$
\left\{\begin{array}{l}
u_{t}(x, t)=\left(k(u(x, t)) u_{x}(x, t)\right)_{x}, \quad(x, t) \in \Omega_{T}, \\
u(x, 0)=g(x), \quad 0<x<1, \\
u(0, t)=\psi_{0}, \quad u(1, t)=f(t), \quad 0<t<T,
\end{array}\right.
$$

where $\Omega_{T}=\left\{(x, t) \in R^{2}: 0<x<1,0<t \leq T\right\}$. The left boundary value $\psi_{0}$ is assumed to be constant. The functions $c_{1}>k(u(x, t)) \geq c_{0}>0$ and $g(x)$ satisfy the following conditions:

(C1) $\left|k_{u}\left(u_{1}\right)-k_{u}\left(u_{2}\right)\right|<d\left|u_{1}-u_{2}\right|$;

(C2) $g(x) \in C^{2}[0,1], g(0)=\psi_{0}, g(1)=f(0)$.

The initial boundary value problem (1) has a unique solution $u(x, t)$ satisfying $u(x, t) \in$ $H^{2,2}[0,1] \cap H^{1,2}[0,1][1-4]$.

In physics, many applications of this problem can be found. The simple model of flame propagation and the spread of biological populations, where $u=u(x, t)$ denotes the temperature and density respectively, are given by the equation in the problem (1). Especially

\section{Springer}

(c) 2013 Ozbilge; licensee Springer. This is an Open Access article distributed under the terms of the Creative Commons Attribution License (http://creativecommons.org/licenses/by/2.0), which permits unrestricted use, distribution, and reproduction in any medium, provided the original work is properly cited. 
$k=k(u(x, t))$ represents the density-dependent coefficient in the problems of the spread of biological populations [5-9].

We consider the inverse problems [10] of determining boundary $u(1, t)$ at $x=1$ in the problem (1) from Dirichlet type of measured output data at the boundaries $x=x_{0}$

$$
u\left(x_{0}, t\right)=\psi_{1}, \quad t \in(0, T]
$$

and from Neumann type of measured output data at the boundaries $x=x_{0}$

$$
u_{x}\left(x_{0}, t\right)=\psi_{2}, \quad t \in(0, T]
$$

Here $u=u(x, t)$ is the solution of the parabolic problem (1). In this context, the parabolic problem (1) will be referred to as a direct (forward) problem with the inputs $g(x), k(u(x, t))$ and $f(t)$. It is assumed that the functions $u\left(x_{0}, t\right)=\psi_{1}$ and $u_{x}\left(x_{0}, t\right)=\psi_{2}$ respectively satisfy the consistency conditions $\psi_{1}=g\left(x_{0}\right)$ and $\psi_{2}=g^{\prime}\left(x_{0}\right)$.

The semigroup approach [11] for inverse problems for the identification of an unknown coefficient in a quasi-linear parabolic equation was studied by Demir and Ozbilge [12]. The study in this paper is based on the philosophy similar to that in [12-15].

The paper is organized as follows. In Section 2, the analysis of the semigroup approach is given for the inverse problem with the single measured output data $u\left(x_{0}, t\right)=\psi_{1}$ given at $x=x_{0}$. The similar analysis is applied to the inverse problem with the single measured output data $u_{x}\left(x_{0}, t\right)=\psi_{2}$ given at the point $x=x_{0}$ in Section 3. Some concluding remarks are given in Section 4.

\section{Analysis of the inverse problem of the boundary condition by Dirichlet type of over measured data $u\left(x_{0}, t\right)=\psi_{1}$}

Consider now the inverse problem with one measured output data $u\left(x_{0}, t\right)=\psi_{1}$ at $x=x_{0}$. In order to formulate the solution of the parabolic problem (1) in terms of a semigroup, let us first arrange the parabolic equation as follows:

$$
u_{t}(x, t)-\left(k(u(0,0)) u_{x}(x, t)\right)_{x}=\left([k(u)-k(u(0,0))] u_{x}(x, t)\right)_{x}, \quad(x, t) \in \Omega_{T}
$$

Then the initial boundary value problem (1) can be rewritten in the following form:

$$
\begin{aligned}
& u_{t}(x, t)-k(u(0,0)) u_{x x}(x, t)=\left((k(u)-k(u(0,0))) u_{x}(x, t)\right)_{x}, \quad(x, t) \in \Omega_{T}, \\
& u(x, 0)=g(x), \quad 0<x<1, \\
& u(0, t)=\psi_{0}, \quad u(1, t)=f(t), \quad 0<t<T .
\end{aligned}
$$

In order to determine the unknown boundary condition $u(1, t)=f(t)$, we need to determine the solution of the following parabolic problem:

$$
\begin{aligned}
& u_{t}(x, t)-k(u(0,0)) u_{x x}(x, t)=\left((k(u)-k(u(0,0))) u_{x}(x, t)\right)_{x}, \quad(x, t) \in \Omega_{T_{0}}, \\
& u(x, 0)=g(x), \quad 0<x<x_{0}, \\
& u(0, t)=\psi_{0}, \quad u\left(x_{0}, t\right)=\psi_{1}, \quad 0<t<T,
\end{aligned}
$$


where $\Omega_{T_{0}}=\left\{(x, t) \in R^{2}: 0<x<x_{0}, 0<t \leq T\right\}$. To formulate the solution of the above problem in terms of a semigroup, we need to define a new function

$$
v(x, t)=u(x, t)+\frac{\left(\psi_{0}-\psi_{1}\right) x}{x_{0}}-\psi_{0}, \quad x \in\left[0, x_{0}\right],
$$

which satisfies the following parabolic problem:

$$
\begin{aligned}
& v_{t}(x, t)+A[v(x, t)]=\left(\left(k\left(v(x, t)+\psi_{0}-\frac{\left(\psi_{0}-\psi_{1}\right) x}{x_{0}}\right)\right.\right. \\
& \left.\quad-k(u(0,0)))\left(v_{x}(x, t)-\frac{\left(\psi_{0}-\psi_{1}\right)}{x_{0}}\right)\right)_{x}, \quad(x, t) \in \Omega_{T}, \\
& v(x, 0)=g(x)+\frac{\left(\psi_{0}-\psi_{1}\right) x}{x_{0}}-\psi_{0}, \quad 0<x<x_{0}, \\
& v(0, t)=0, \quad v\left(x_{0}, t\right)=0, \quad 0<t<T .
\end{aligned}
$$

Here, $A[\cdot]:=-k(u(0,0)) \frac{\left.d^{2} \cdot \cdot\right]}{d x^{2}}$ is a second-order differential operator and its domain is $D_{A}=\left\{v(x) \in H_{0}^{2,2}(0,1) \cap H_{0}^{3,2}[0,1]: v(0)=0=v(1)\right\}$, where $H_{0}^{2,2}(0,1)=\overline{C_{0}^{2}(0,1)}$ and $H_{0}^{1,2}[0,1]=\overline{C_{0}^{1}[0,1]}$ are Sobolev spaces. Obviously, by completion $g(x) \in D_{A}$, since the initial value function $g(x)$ belongs to $C^{3}[0,1]$. Hence, $D_{A}$ is dense in $H_{0}^{2,2}[0,1]$, which is a necessary condition for being an infinitesimal generator.

In the following, despite doing the calculations in the smooth function space, by completion they are valid in the Sobolev space.

Let us denote the semigroup of linear operators by $T(t)$ generated by the operator $A$ $[8,9]$. We can easily find the eigenvalues and eigenfunctions of the differential operator $A$. Moreover, the semigroup $T(t)$ can be easily constructed by using the eigenvalues and eigenfunctions of the infinitesimal generator $A$. Hence, we first consider the following eigenvalue problem:

$$
\begin{aligned}
& A \phi(x)=\lambda \phi(x), \\
& \phi(0)=0 ; \quad \phi\left(x_{0}\right)=0 .
\end{aligned}
$$

We can easily determine that the eigenvalues are $\lambda_{n}=k(u(0,0)) \frac{n^{2} \pi^{2}}{x_{0}^{2}}$ for all $n=1, \ldots$ and the corresponding eigenfunctions are $\phi_{n}(x)=\sin \left(\frac{n \pi x}{x_{0}}\right)$. In this case, the semigroup $T(t)$ can be represented in the following way:

$$
T(t) U(x, s)=\sum_{n=0}^{\infty}\left\langle\phi_{n}(x), U(x, s)\right| e^{-\lambda_{n} t} \phi_{n}(x),
$$

where $\left\langle\phi_{n}(x), U(x, s)\right\rangle=\int_{0}^{1} \phi_{n}(x) U(x, s) d x$. Under this representation, the null space of the semigroup $T(t)$ of the linear operators can be defined as follows:

$$
N(T)=\left\{U(x, s):\left\langle\phi_{n}(x), U(x, s)\right\rangle=0, \text { for all } n=0,1,2,3, \ldots\right\} .
$$

From the definition of the semigroup $T(t)$, we can say that the null space of it consists of only zero function, i.e., $N(T)=\{0\}$. This result is very important for the uniqueness of the unknown boundary condition $u(1, t)$. 
The unique solution of the initial-boundary value problem (7) in terms of the semigroup $T(t)$ can be represented in the following form:

$$
\begin{aligned}
v(x, t)= & T(t) v(x, 0)+\int_{0}^{t} T(t-s)\left(\left(k\left(v(x, s)+\psi_{0}-\frac{\left(\psi_{0}-\psi_{1}\right) x}{x_{0}}\right)\right.\right. \\
& \left.-k(u(0,0)))\left(v_{x}(x, s)-\frac{\left(\psi_{0}-\psi_{1}\right)}{x_{0}}\right)\right)_{x} d s .
\end{aligned}
$$

Now, by using the identity (6) and taking the initial value $u(x, 0)=g(x)$ into account, the integral equation for the solution $u(x, t)$ of the parabolic problem (5) in terms of a semigroup can be written in the following form:

$$
\begin{aligned}
u(x, t)= & \psi_{0}-\frac{\left(\psi_{0}-\psi_{1}\right) x}{x_{0}}+T(t)\left(g(x)+\frac{\left(\psi_{0}-\psi_{1}\right) x}{x_{0}}-\psi_{0}\right) \\
& +\int_{0}^{t} T(t-s)\left((k(u(x, s))-k(u(0,0))) u_{x}(x, s)\right)_{x} d s .
\end{aligned}
$$

In order to arrange the above integral equation, let us define the following:

$$
\begin{aligned}
& \zeta(x)=\left(g(x)+\frac{\left(\psi_{0}-\psi_{1}\right) x}{x_{0}}-\psi_{0}\right), \\
& \xi(x, t)=\left((k(u(x, t))-k(u(0,0))) u_{x}(x, t)\right)_{x} .
\end{aligned}
$$

Then we can rewrite the integral equation in terms of $\zeta(x)$ and $\xi(x, s)$ in the following form:

$$
u(x, t)=\psi_{0}-\frac{\left(\psi_{0}-\psi_{1}\right) x}{x_{0}}+(T(t) \zeta(\cdot))(x, t)+\int_{0}^{t}(T(t-s) \xi(\cdot, s))(x, t, s) d s .
$$

This is the integral representation of a solution of the initial-boundary value problem (5) on $\Omega_{T_{0}}=\left\{(x, t) \in R^{2}: 0<x<x_{0}, 0<t \leq T\right\}$. It is obvious from the eigenfunctions $\phi_{n}(x)$, the domain of eigenfunctions can be extended to the closed interval $[0,1]$. Moreover they are continuous on $[0,1]$. Under this extension, the uniqueness of the solutions of the initialboundary value problems (4) and (5) imply that the integral representation (11) becomes the integral representation of a solution of the initial-boundary value problem (4) on $\Omega_{T}=$ $\left\{(x, t) \in R^{2}: 0<x<1,0<t \leq T\right\}$.

At this stage, it is obvious that the solution of the inverse problem can easily be obtained by substituting $x=1$ into the integral representation (11) of the solution $u(x, t)$,

$$
u(1, t)=f(t)=\psi_{0}-\frac{\left(\psi_{0}-\psi_{1}\right)}{x_{0}}+(T(t) \zeta(\cdot))(1, t)+\int_{0}^{t}(T(t-s) \xi(\cdot, s))(1, t, s) d s,
$$

which implies that $f(t)$ can be determined analytically.

The right-hand side of the identity (12) defines the semigroup representation of the unknown boundary condition $u(1, t)$ at $x=1$.

\section{Analysis of the inverse problem of the boundary condition by Neumann type of over measured data $u_{x}\left(x_{0}, t\right)=\psi_{2}$}

Consider now the inverse problem with one measured output data $u_{x}\left(x_{0}, t\right)=\psi_{2}$ at $x=x_{0}$. In order to formulate the solution of the parabolic problem (1) in terms of a semigroup, 
we arrange the parabolic equation as follows:

$$
u_{t}(x, t)-\left(k(u(0,0)) u_{x}(x, t)\right)_{x}=\left([k(u)-k(u(0,0))] u_{x}(x, t)\right)_{x^{\prime}} \quad(x, t) \in \Omega_{T} .
$$

Then the initial boundary value problem (1) can be rewritten in the following form:

$$
\begin{aligned}
& u_{t}(x, t)-k(u(0,0)) u_{x x}(x, t)=\left((k(u)-k(u(0,0))) u_{x}(x, t)\right)_{x}, \quad(x, t) \in \Omega_{T}, \\
& u(x, 0)=g(x), \quad 0<x<1, \\
& u(0, t)=\psi_{0}, \quad u(1, t)=f(t), \quad 0<t<T .
\end{aligned}
$$

In order to determine the unknown boundary condition $u(1, t)=f(t)$, we need to determine the solution of the following parabolic problem:

$$
\begin{aligned}
& u_{t}(x, t)-k(u(0,0)) u_{x x}(x, t)=\left((k(u)-k(u(0,0))) u_{x}(x, t)\right)_{x}, \quad(x, t) \in \Omega_{T_{0}}, \\
& u(x, 0)=g(x), \quad 0<x<x_{0}, \\
& u(0, t)=\psi_{0}, \quad u_{x}\left(x_{0}, t\right)=\psi_{2}, \quad 0<t<T,
\end{aligned}
$$

where $\Omega_{T_{0}}=\left\{(x, t) \in R^{2}: 0<x<x_{0}, 0<t \leq T\right\}$.

To formulate the solution of the above problem in terms of a semigroup, we need to define a new function

$$
v(x, t)=u(x, t)-\psi_{2} x-\psi_{0}, \quad x \in\left[0, x_{0}\right]
$$

which satisfies the following parabolic problem:

$$
\begin{aligned}
& v_{t}(x, t)+B[v(x, t)]=\left(\left(k\left(v(x, t)+\psi_{0}+\psi_{2} x\right)-k(u(0,0))\right)\left(v_{x}(x, t)+\psi_{2}\right)\right)_{x^{\prime}} \\
& \quad(x, t) \in \Omega_{T}, \\
& v(x, 0)=g(x)-\psi_{2} x-\psi_{0}, \quad 0<x<x_{0}, \\
& v(0, t)=0, \quad v_{x}\left(x_{0}, t\right)=0, \quad 0<t<T .
\end{aligned}
$$

Here $B[\cdot]:=-k(u(0,0)) \frac{d^{2}[\cdot]}{d x^{2}}$ is a second-order differential operator, its domain is $D_{B}=$ $\left\{v \in C^{2}\left(0, x_{0}\right) \cap C^{1}\left[0, x_{0}\right]: v(0)=v_{x}\left(x_{0}\right)=0\right\}$. It is clear from the definition of $D_{B}$ that $D_{B} \subset$ $C^{2}\left[0, x_{0}\right]$.

Let us denote the semigroup of linear operators by $S(t)$ generated by the operator $B$ $[8,9]$. We can easily find the eigenvalues and eigenfunctions of the differential operator $B$. Moreover, the semigroup $S(t)$ can be easily constructed by using the eigenvalues and eigenfunctions of the infinitesimal generator $B$. Hence, we first consider the following eigenvalue problem:

$$
\begin{aligned}
& B \phi(x)=\lambda \phi(x), \\
& \phi(0)=0 ; \quad \phi_{x}\left(x_{0}\right)=0 .
\end{aligned}
$$

We can easily determine that the eigenvalues are $\lambda_{n}=k(u(0,0)) \frac{(2 n+1)^{2} \pi^{2}}{4 x_{0}^{2}}$ for all $n=0,1, \ldots$ and the corresponding eigenfunctions are $\phi_{n}(x)=\sin \left(\frac{(2 n+1) \pi x}{2 x_{0}}\right)$. In this case, the semigroup 
$S(t)$ can be represented in the following way:

$$
S(t) U(x, s)=\sum_{n=0}^{\infty}\left\langle\phi_{n}(x), U(x, s)\right\rangle e^{-\lambda_{n} t} \phi_{n}(x),
$$

where $\left\langle\phi_{n}(x), U(x, s)\right\rangle=\int_{0}^{1} \phi_{n}(x) U(x, s) d x$. Under this representation, the null space of the semigroup $S(t)$ of the linear operators can be defined as follows:

$$
N(S)=\left\{U(x, s):\left\langle\phi_{n}(x), U(x, s)\right\rangle=0, \text { for all } n=0,1,2,3, \ldots\right\} .
$$

From the definition of the semigroup $S(t)$, we can say that the null space of it consists of only zero function, i.e., $N(S)=\{0\}$. This result is very important for the uniqueness of the unknown boundary condition $u(1, t)$.

The unique solution of the initial-boundary value problem (16) in terms of the semigroup $S(t)$ can be represented in the following form:

$$
\begin{aligned}
v(x, t)= & S(t) v(x, 0) \\
& +\int_{0}^{t} S(t-s)\left(\left(k\left(v(x, s)+\psi_{0}+\psi_{2} x\right)-k(u(0,0))\right)\left(v_{x}(x, s)+\psi_{2}\right)\right)_{x} d s .
\end{aligned}
$$

Now, by using the identity (15) and taking the initial value $u(x, 0)=g(x)$ into account, the integral equation for the solution $u(x, t)$ of the parabolic problem (14) in terms of a semigroup can be written in the following form:

$$
\begin{aligned}
u(x, t)= & \psi_{0}+\psi_{2} x+S(t)\left(g(x)-\psi_{0}-\psi_{2} x\right) \\
& +\int_{0}^{t} S(t-s)\left((k(u(x, s))-k(u(0,0))) u_{x}(x, s)\right)_{x} d s
\end{aligned}
$$

In order to arrange the above integral equation, let us define the following:

$$
\begin{aligned}
& \zeta(x)=\left(g(x)-\psi_{0}-\psi_{2} x\right), \\
& \xi(x, t)=\left((k(u(x, t))-k(u(0,0))) u_{x}(x, t)\right)_{x} .
\end{aligned}
$$

Then we can rewrite the integral equation in terms of $\zeta(x)$ and $\xi(x, s)$ in the following form:

$$
u(x, t)=\psi_{0}+\psi_{2} x+(S(t) \zeta(\cdot))(x, t)+\int_{0}^{t}(S(t-s) \xi(\cdot, s))(x, t, s) d s
$$

This is the integral representation of a solution of the initial-boundary value problem (14) on $\Omega_{T_{0}}=\left\{(x, t) \in R^{2}: 0<x<x_{0}, 0<t \leq T\right\}$. It is obvious from the eigenfunctions $\phi_{n}(x)$, the domain of eigenfunctions can be extended to the closed interval $[0,1]$. Moreover, they are continuous on $[0,1]$. Under this extension, the uniqueness of the solutions of the initialboundary value problems (13) and (14) imply that the integral representation (20) becomes the integral representation of a solution of the initial-boundary value problem (13) on $\Omega_{T}=\left\{(x, t) \in R^{2}: 0<x<1,0<t \leq T\right\}$. 
Substituting $x=1$ into the integral representation (20) of the solution $u(x, t)$ yields

$$
u(1, t)=f(t)=\psi_{0}+\psi_{2}+(S(t) \zeta(\cdot))(1, t)+\int_{0}^{t}(S(t-s) \xi(\cdot, s))(1, t, s) d s
$$

which implies that $f(t)$ can be determined analytically.

The right-hand side of the identity (21) defines the semigroup representation of the unknown boundary condition $u(1, t)$ at $x=1$...

\section{Conclusion}

The goal of this study is to identify the unknown boundary condition $u(1, t)$ at $x=1$ by using the over measured data $u\left(x_{0}, t\right)=\psi_{1}$ and $u_{x}\left(x_{0}, t\right)=\psi_{2}$. The key point here is the unique extensions of solutions on $\left[0, x_{0}\right]$ to the closed interval $[0,1]$ which are implied by the uniqueness of the solutions. This key point leads to the integral representation of the unknown boundary condition $u(1, t)$ at $x=1$ obtained analytically....

\section{Competing interests}

The author declares that they have no competing interests.

\section{Acknowledgements}

Dedicated to my father and mother Yusuf/Sevim Ozbilge.

The research was supported by parts by the Scientific and Technical Research Council (TUBITAK) of Turkey and Izmir University of Economics....

Received: 23 November 2012 Accepted: 17 December 2012 Published: 4 January 2013

\section{References}

1. DuChateau, P: Monotonicity and invertibility of coefficient-to-data mappings for parabolic inverse problems. SIAM J. Math. Anal. 26, 1473-1487 (1995)

2. Isakov, V: On uniqueness in inverse problems for quasilinear parabolic equations. Arch. Ration. Mech. Anal. 124, 1-13 (1993)

3. Pilant, MS, Rundell, W: A uniqueness theorem for conductivity from overspecified boundary data. J. Math. Anal. Appl. 136, 20-28 (1988)

4. Renardy, M, Rogers, R: An Introduction to Partial Differential Equations. Springer, New York (2004)

5. Cannon, JR: The One-Dimensional Heat Equation. Addison-Wesley, Reading (1984)

6. DuChateau, $P$, Thelwell, R, Butters, G: Analysis of an adjoint problem approach to the identification of an unknown diffusion coefficient. Inverse Probl. 20, 601-625 (2004)

7. Showalter, R: Monotone Operators in Banach Spaces and Nonlinear Partial Differential Equations. Am. Math. Soc, Providence (1997)

8. Hasanov, A, Demir, A, Erdem, A: Monotonicity of input-output mappings in inverse coefficient and source problem for parabolic equations. J. Math. Anal. Appl. 335, 1434-1451 (2007)

9. Hasanov, A, DuChateau, P, Pektas, B: An adjoint approach and coarse-fine mesh method for identification of the diffusion coefficient in a linear parabolic equations. J. Inverse III-Posed Probl. 14, 435-463 (2006)

10. DuChateau, P, Gottlieb, J: Introduction to Inverse Problems in Partial Differential Equations for Engineers, Physicists and Mathematicians. Kluwer Academic, Netherlands (1996)

11. Ashyralyev, A, San, ME: An approximation of semigroup method for stochastic parabolic equations. Abstr. Appl. Anal. 2012, 684248 (2012). doi:10.1155/2012/684248

12. Demir, A, Ozbilge, E: Semigroup approach for identification of the unknown diffusion coefficient in a quasi-linear parabolic equation. Math. Methods Appl. Sci. 30, 1283-1294 (2007)

13. Ozbilge, E: Identification of the unknown diffusion coefficient in a quasi-linear parabolic equation by semigroup approach with mixed boundary conditions. Math. Methods Appl. Sci. 31, 1333-1344 (2008)

14. Demir, A, Ozbilge, E: Analysis of a semigroup approach in the inverse problem of identifying an unknown coefficient. Math. Methods Appl. Sci. 31, 1635-1645 (2008)

15. Demir, A, Hasanov, A: Identification of the unknown diffusion coefficient in a linear parabolic equation by the semigroup approach. J. Math. Anal. Appl. 340, 5-15 (2008)

doi:10.1186/1687-2770-2013-2

Cite this article as: Ozbilge: Determination of the unknown boundary condition of the inverse parabolic problems via semigroup method. Boundary Value Problems 2013 2013:2. 